\title{
The phase of the de Haas-van Alphen oscillations, the Berry phase, and band-contact lines in metals
}

\author{
G.P. Mikitik and Yu.V. Sharlai \\ B. Verkin Institute for Low Temperature Physics and Engineering of the National Academy of Sciences of Ukraine \\ 47 Lenin Ave., Kharkov 61103, Ukraine \\ E-mail: mikitik@ilt.kharkov.ua
}

Received June 15, 2006

\begin{abstract}
We point out that measurements of a phase of the de Haas-van Alphen oscillations can give information on a degeneracy of electron-energy bands in a metal even though this degeneracy occurs far away from its Fermi level. As an illustration of this statement, the published experimental data on the de Haas-van Alphen effect in LaRhIn 5 , graphite, and zinc are discussed.
\end{abstract}

PACS: 71.18.+y Fermi surface: calculations and measurements; effective mass, $g$ factor; 03.65.Vf Phases: geometric; dynamic or topological.

Keywords: Berry phase, de Haas-van Alphen oscillations, degeneracy of electron-energy bands.

As it is well known [1], a frequency of de Haas-van Alphen oscillations, or of other oscillation effects in the magnetic field, gives an extremal cross-section area $S_{\text {ex }}$ of the Fermi surface of a metal. On the other hand, a phase of these oscillations is expressed via the constant $\gamma$ of the semiclassical quantization rule for the energy of a Bloch electron in the magnetic field $H$ [2]. However, this phase is commonly ignored in experiments since it is generally believed $[1,3]$ that $\gamma$ is equal to $1 / 2$ in absence of the magnetic breakdown, and hence the phase does not contain any essential information on the electron-band structures of a metal. In this paper we call attention to the fact that $\gamma$ can differ from its usual value $1 / 2$ if a band-contact line penetrates the extremal cross section. Thus, measurement of the phase of the de Haas-van Alphen oscillations can provide an unique information on the band degeneracy even though this degeneracy occurs far away from the Fermi level.

In the magnetic field a semiclassical electron orbit in the space of wave vectors $\mathbf{k}$ (i.e., in the Brillouin zone of a metal) is the intersection of the constant-energy surface, $\varepsilon(\mathbf{k})=$ const, with the plane, $k_{H}=$ const, where $k_{H}$ is the component of $\mathbf{k}$ along the magnetic field $\mathbf{H}$. In the case of the closed orbit the quantization condition for energy levels of the electron looks like $[1,2]$ :

$$
S\left(\varepsilon, k_{H}\right)=\frac{2 \pi e H}{\hbar c}(n+\gamma),
$$

where $S$ is the cross-sectional area of the closed orbit in the k space; $n$ is a large integer $(n>0)$; $e$ is the absolute value of the electron charge. If the magnetic breakdown is negligible for the orbit under study, the constant $\gamma$ has the universal value [4]:

$$
\gamma=\frac{1}{2}
$$

It is this value that is commonly used in describing oscillation phenomena in metals [1]. For simplicity, in Eq. (1) we neglect the spin of the electron. Effects of the spin will be briefly discussed at the end of the paper.

In recent years the concept of the so-called Berry phase [5] has attracted considerable attention thanks to its fundamental origin, see, e.g., Refs. 6, 7 and citation therein. According to Berry, if a Hamiltonian of a quantum system depends on parameters, and if the parameters undergo adiabatic changes so that they eventually return to their original values, the wave function of the system can acquire the so-called geometrical phase in addition to the familiar dynamical one. This additional phase (the Berry phase) differs from zero when the trajectory $\Gamma$ of the system in the parameter space is located near a point at which the states of the system are degenerate [5]. In analyzing this situation, Berry assumed that the Hamiltonian of the system is a Hermitian matrix which is linear in deviations of the parameters from the point, and he presented his final result in the pictorial form. He found that such the point 
can be considered as a «monopole» in the parameter space when the geometrical phase is calculated. In other words, the point «generates» a field which coincides in the form with that of the monopole, and the flux of this Berry field through the contour $\Gamma$ gives the geometrical phase of the system.

Berry's result is applicable to the semiclassical electron trajectories in crystals, with the Brillouin zone playing the role of the parameter space [8]. However, in crystals with the inversion symmetry and a weak spin-orbit interaction, the Berry phase of the electrons has the specific features [9] which are due to the fact that the electron states are invariant under the simultaneous inversion of time and spatial coordinates. This invariance permits one to transform the Hermitian Hamiltonian of the electron into the real form for any point of the Brillouin zone. As a consequence, the character of the energy-band degeneracy differs from that considered by Berry. Now the electron energy bands $\varepsilon_{l}(\mathbf{k})$ contact along lines in the Brillouin zone rather than at points, and the lines need not be symmetry axes [10]. In other words, Berry's monopoles in the $\mathbf{k}$ space disappear. In this context, it is frequently implied that the Berry phase is equal to zero for electron orbits in such crystals, and that a nonzero Berry phase can occur only in crystals with sufficiently strong spin-orbit interaction (in this case, the nonzero Berry phase is only the other interpretation of a nonzero orbital $g$ factor of the electron). However, as it was shown in our paper [9], a nonzero Berry phase also exists in crystals in which this interaction is negligible. We showed that the above-mentioned band-contact lines play the role of infinitely thin «solenoids» which generate the Berry field with the flux $\pm \pi$, and the situation similar to the Aharonov-Bohm effect [11] occurs: Although this field is zero outside the solenoids, but if the electron orbit surrounds a contact line of its band with some other band, the flux threads the orbit, and the electron acquires the Berry phase $\varphi_{B}= \pm \pi$ when it moves around this line. It is clear that in this case the Berry phase does not depend on the shape and the size of the electron orbit but is specified only by its topological characteristics (there is a linking of the orbit with the band-contact line or not).

The Berry phase of the electron modifies the constant $\gamma$ in the semiclassical quantization rule. The constant $\gamma$ is now given by the formula [9]:

$$
\gamma=\frac{1}{2}-\frac{\Phi_{B}}{2 \pi} .
$$

The meaning of formula (3) is the following: When the electron makes a complete circuit in its orbit, the change of the phase of its wave function consists of the usual semiclassical part $\hbar c S / e H$, the shift $-\pi$ associated with the so-called turning points of the orbit where the semiclassical approximation fails, and the Berry phase. Equat- ing this change to $2 \pi n$, one arrives at Eqs. (1), (3). Thus, when the electron orbit links to the band-contact line, one obtains $\gamma=0$ (the values $\gamma=0$ and $\gamma=1$ are equivalent) instead of the usual value $\gamma=1 / 2$.

As was mentioned above, the constant $\gamma$ specifies the phase of the de Haas-van Alphen oscillations. For example, the first harmonic of the de Haas-van Alphen oscillations of the magnetic susceptibility has the form [2],

$$
\chi \cos \left(2 \pi \frac{\nu}{H}+\varphi\right),
$$

where $v=\hbar c S_{\text {ex }} /(2 \pi e), S_{\text {ex }}$ is some extremal cross section of the Fermi surface of a metal in $k_{H}$, a positive $\chi$ is the amplitude of this first harmonic, and $\varphi$ is its phase which is given by

$$
\varphi=-2 \pi \gamma+\delta
$$

with $\delta= \pm \pi / 4$ for a minimum and maximum cross-section $S_{\text {ex }}$, respectively (and $\delta=0$ in the case of a two-dimensional Fermi surface [12]). The phase $\varphi$ (together with the frequency) can be found by the Fourier analysis of the magnetic-susceptibility oscillations [12]. Thus, measurements of the phase $\varphi$ for the first harmonic enable one to find $\gamma$.

If at low temperatures many harmonics contribute to the oscillating part $M$ of the magnetization, $\gamma$ can be found even without the Fourier analysis. In this case it is possible to take the summation over the harmonics in the Lifshitz-Kosevich formula for $M$. Using results of Ref. 13, we then arrive at

$$
M=-\frac{e^{3 / 2} \hbar^{1 / 2}}{\pi^{5 / 2} c^{3 / 2}} H^{1 / 2} \frac{S_{\mathrm{ex}}}{\left|m^{*}\right|}\left|\frac{\partial^{2} S}{\partial k_{H}^{2}}\right|^{-1 / 2} \times \widetilde{M},
$$

where $m *$ is the cyclotron mass of the electron, and

$$
\tilde{M}=\zeta\left(-\frac{1}{2},\left\{\frac{c \hbar S_{\mathrm{ex}}}{2 \pi e H}-\gamma\right\}\right)
$$

in the case of the maximum cross section $S_{\text {ex }}$, and

$$
\widetilde{M}=-\zeta\left(-\frac{1}{2}, 1-\left\{\frac{c \hbar S_{\mathrm{ex}}}{2 \pi e H}-\gamma\right\}\right)
$$

for the case of the minimum $S_{\text {ex }}$. Here $\zeta(-1 / 2, z)$ is the generalized Riemann zeta function [13], and $\{z\}$ denotes the fractional part of the number $z$. Equations (7), (8) describe the saw-tooth oscillations with sharp peaks at the magnetic fields $H_{n}$,

$$
\frac{e}{c \hbar} H_{n}=\frac{S_{\mathrm{ex}}}{2 \pi}(n+\gamma)^{-1},
$$

which correspond to the crossing of the $n$th Landau level with the Fermi energy. Note that for the maximum $S_{\text {ex }}$ the peaks are directed upwards, while for the minimum 
$S_{\text {ex }}$ downwards. In this situation it is possible to find $\gamma$ using the procedure of Shoenberg [1]. Plotting measured $1 / H_{n}$ versus $n$ and extrapolating this straight line to the origin of the coordinates, one obtains $\gamma$.

Summing up the above considerations, we can state that measurements of a phase of the de Haas-van Alphen oscillations give $\gamma$ for the appropriate electron-energy band and hence enable one to detect those contact lines of this band which penetrate the extremal cross section of the Fermi surface. We now illustrate this statement by several examples.

In the recent experimental investigation [14] of the de Haas-van Alphen effect in LaRhIn ${ }_{5}$, the oscillations of magnetization associated with a small cross-section of the Fermi surface of this metal were detected. Authors of that paper attributed these oscillations to a small electron pocket of the Fermi surface. The experimental data [14] obtained at a low temperature $(1.5 \mathrm{~K})$ reveal the sharp peaks in the magnetization of $\mathrm{LaRhIn}_{5}$ when the magnetic field $H$ is parallel to the [001] direction of this tetragonal compound, Fig. 1. The analysis of the peak positions gives $\gamma=0$ [15], see inset $(a)$ in Fig. 1, and we conclude that the oscillations in the magnetization result from some small group of charge carriers near the band-contact line. The downward peaks mean that we deal with the situation shown in the second inset of Fig. 1, i.e., with a small «neck» containing a band-contact line rather than with a small electron pocket.

The second example concerns graphite. The electronic spectrum of graphite is described by the SlonzewskiWeiss-McClure model $[16,17]$, and values of the main parameters of this model were found sufficiently accurately from the analysis of various experimental data; see, e.g., the review of Brandt et al. [18] and references

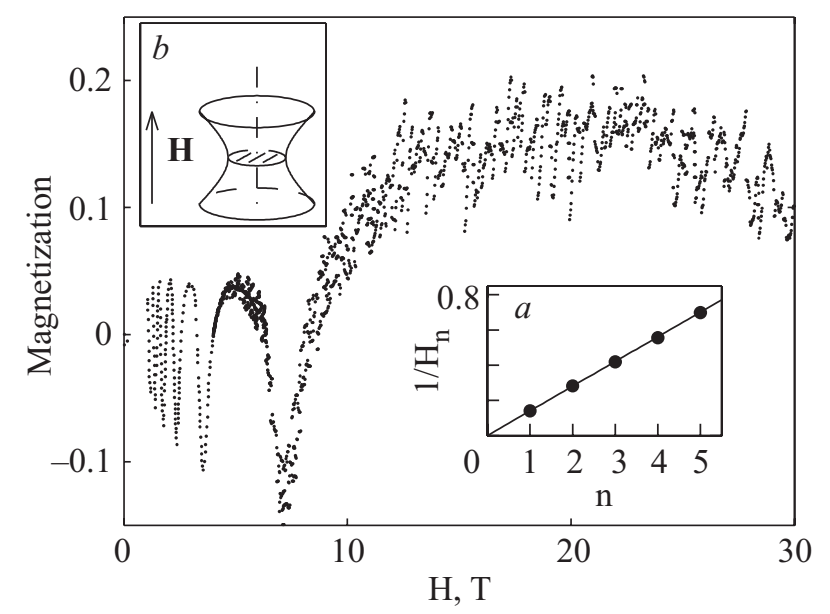

Fig. 1. The experimental data [14] on the magnetization of LaRhIn $_{5}$ (dots). The inset (a) shows the dependence of the experimental values of $1 / H_{n}$ on $n$. This dependence gives $\gamma=0$. The inset $(b)$ shows the Fermi surface of the small group in $\mathrm{LaRhIn}_{5}$, and the band-contact line (the dash-dot line). therein. The Fermi surface of graphite consists of elongated pockets enclosing the edge $H K H$ of its Brillouin zone, see Fig. 2. These pockets are formed by the two majority groups of electrons (e) and holes (h) which are located near the points $K$ and $H$ of the Brillouin zone, respectively. The electron majority is formed by electrons of the third band $\varepsilon_{3}(\mathbf{k})$, while the hole majority belongs to the second band $\varepsilon_{2}(\mathbf{k})$.

As it is well known [18], in the edge $H K H$ of the Brillouin zone of graphite the bands $\varepsilon_{2}(\mathbf{k})$ and $\varepsilon_{3}(\mathbf{k})$ are degenerate, and in a small vicinity of the edge these bands split linearly in a deviation of the wave vector $\mathbf{k}$ from the edge. In other words, the edge is the band-contact line, and one might expect to find $\gamma=0$ for the orbits surrounding this axis. However, the experimental data of Refs. 19, 20 show that at the magnetic field $H$ directed along the $H K H$ axis, one has the usual value $\gamma=1 / 2$ for the maximum cross sections of the electron and hole majorities. Thus, we conclude that an even number of the band-contact lines have to penetrate these cross sections (although each line adds 0.5 to $\gamma$, but within the semiclassical approximation $\gamma$ is defined up to an integer). The recent analysis of the Slonzewski-Weiss-McClure model [21] does show that near the edge $H K H$ there are three additional contact lines of the same bands, and thus, both these cross sections are penetrated by the four band-contact lines, see Fig. 2.
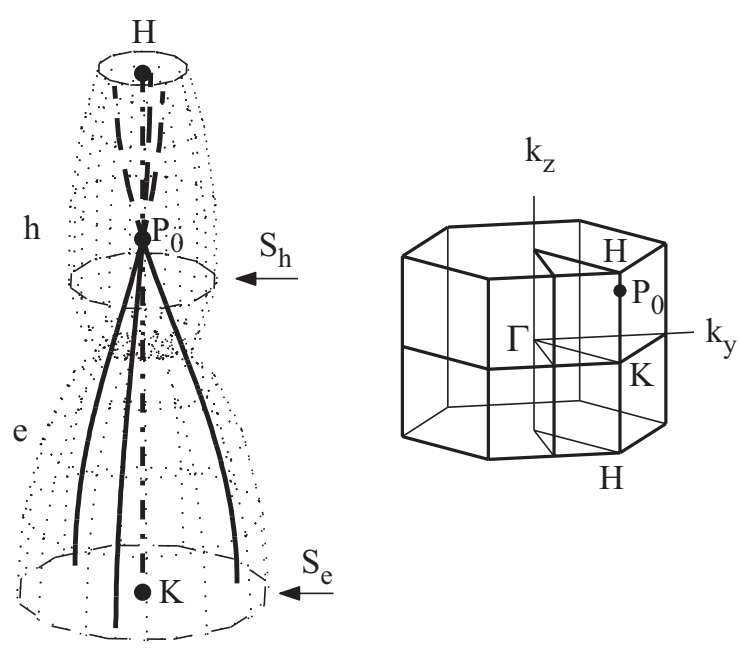

Fig. 2. Sketch of the Fermi surface (a half of it) and of the band contact lines in graphite. The accidental contact of the bands $\varepsilon_{2}(\mathbf{k})$ and $\varepsilon_{3}(\mathbf{k})$ occurs along the solid lines, while the dashed lines mark the accidental contact of the bands $\varepsilon_{3}(\mathbf{k})$ and $\varepsilon_{4}(\mathbf{k})$. The same bands are in contact along the $H K H$ axis due to the symmetry of the crystal. All the lines merge at the point $P_{0}$ that is very close to the point $H\left(H P_{0} \approx 0.01 H K\right)$. Shown are also the maximum cross sections of the electron $\left(S_{e}\right)$ and hole $\left(S_{h}\right)$ majorities for the magnetic field along the $H K H$ axis. On the right, the Brillouin zone of graphite is shown. 
Before considering the third example, let us discuss the effect of the spin-orbit interaction on the quantization rule and the band degeneracy [but we shall still neglect the direct spin contribution to Eq. (1)]. With this interaction, the semiclassical quantization rule (1) is modifies as follows [1]:

$$
S\left(\varepsilon, k_{z}\right)=\frac{2 \pi e H}{\hbar c}\left(n+\frac{1}{2} \pm \delta\right),
$$

where $\delta=g m * / 4 m, m^{*}$ and $m$ are the cyclotron and the electron masses, and $g$ is the so-called orbital $g$ factor of the electron orbit. The spin-orbit interaction also lifts the accidental degeneracy of the bands (which is not due to the symmetry of the crystal). But if this interaction is not too strong so that the spin-orbit splitting of the two degenerate bands is essentially smaller than energy gaps on the electron trajectory between the band under study and other bands of the metal, the concept of the band-contact line is still valid approximately. As it was shown in our paper [22], if the semiclassical electron orbit in the magnetic field surrounds such a split band-contact line, one has $g \approx 2 m / m^{*}, \delta \approx 1 / 2$, and formula (10) is equivalent to Eq. (1) with $\gamma=0$. In other words, expression (3) is robust to «switching on» the spin-orbit interaction. We emphasize that in this situation the $g$ factor is large even for a very weak spin-orbit interaction, and this result is the equivalent description of the nonzero Berry phase (instead of $\gamma=0$ ).

We now discuss the third example, the electron orbits on the so-called needle of zinc. The Brillouin zone of zinc coincides with that of graphite, see Fig. 2, and the needle is located in the vicinity of the point $K$. Near this point the electron-band structure of zinc is described by the Bennett-Falicov model [23]. According to this model, there are three electron-energy bands which are close to each other in the vicinity of the point $K$. The electrons of the third (uppermost) band just form the needle. One of the two energy gaps at the point $K$ is formed by the spin-orbit interaction, while the other gap is caused by the crystal potential. Both these gaps are of the same order and small $(\sim 1-3 \mathrm{mRy})$.

In the framework of this model two sets of its parameters were found [24] that reproduce the known experimental data on the de Haas-van Alphen effect in zinc [25-27]. These two sets correspond to the energy spectra that differ in number of the split band-contact lines [28]. The first set leads to a single contact line of the third and second bands, and this line coincides with the $H K H$ axis, while the second set gives the four contact lines of the same bands, see the insets in Fig. 3. If the Fermi level $\zeta$ is considerably above the edge of the third band, the energy gaps on the electron trajectory are large, and according to the above result for $\delta$, this $\delta$ is completely determined by number of the band-contact lines: For the case of the single line, $\delta$ is a

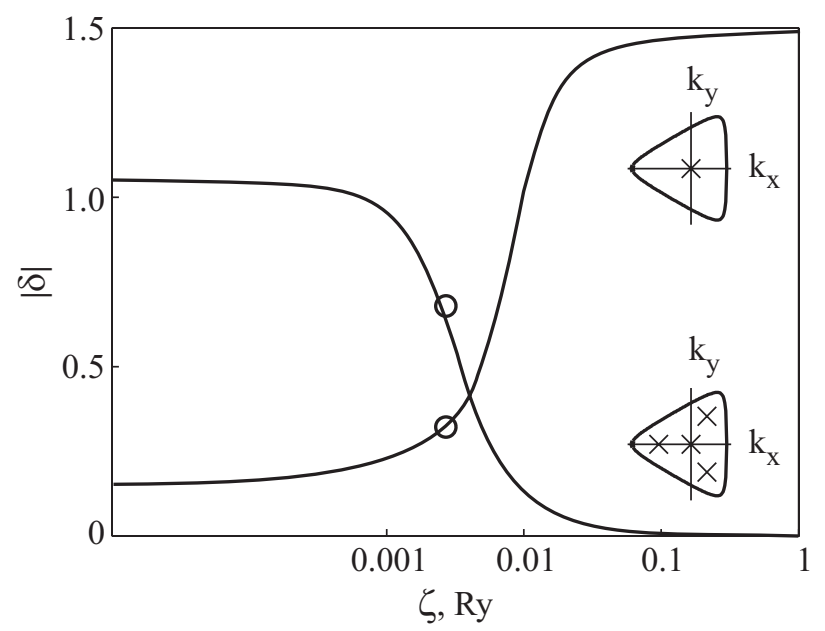

Fig. 3. The dependence of the quantity $\delta \equiv g m * / 4 m$ on the Fermi energy $\zeta$ measured from the edge of the third band whose electrons forms the needle in zinc. Here $g$ and $m^{*}$ are the $g$ factor and the cyclotron mass of the extremal orbit on the needle when the magnetic field is along the $k_{z}$ axis (see Fig. 2); $m$ is the electron mass. The two curves give $\delta(\zeta)$ for the two sets [24] of the parameters in the Bennett-Falicov model [23]. The insets near the curves show the positions of the band-contact lines in these two cases. The circles mark the values of $\delta$ experimentally obtained for zinc [25].

half-integer, while for the case of the four lines, one has an integer $\delta$, and these values of $\delta$ differ by $3 / 2$. These considerations agree with Fig. 3 where we show the dependence of $\delta$ on $\zeta$ calculated in Ref. 28 for the abovementioned two sets of the parameters at magnetic fields when the magnetic breakdown is negligible. Note that under the normal conditions the Fermi energy of zinc is not far from the edge of the third band, the $g$ factor is thus affected by the spin-orbit interaction, and $\delta$ differs from the limiting values. However, the data of Fig. 3 suggest that investigations [29] of the pressure dependence of $\delta$ can, in principle, enable one to distinguish between the two sets of the parameters, and thus to find the number of the band-contact lines inside the needle of zinc.

1. D. Shoenberg, Magnetic Oscillations in Metals, Cambridge University Press, Cambridge, England (1984).

2. I.M. Lifshits and A.M. Kosevich, Zh. Eksp. Teor. Fiz. 29, 730 (1955) [Sov. Phys. JETP 2, 636 (1956)].

3. E.M. Lifshitz and L.P. Pitaevskii, Statistical Physics, Pergamon Press, Oxford (1986), Pt.2.

4. G.E. Zilberman, Zh. Eksp. Teor. Fiz. 32, 296 (1957) [Sov. Phys. JETP 5, 208 (1957)].

5. M.V. Berry, Proc. Roy. Soc. London A392, 45 (1984).

6. A.Shapere and F. Wilczek, Geometrical Phase in Physics, World Scientific, Singapore (1989).

7. R. Resta, J. Phys.: Condens Matter 12, R107 (2000).

8. J. Zak, Phys. Rev. Lett. 62, 2747 (1989).

9. G.P. Mikitik and Yu.V. Sharlai, Phys. Rev. Lett. 82, 2147 (1999). 
10. C. Herring, Phys. Rev. 52, 365 (1937).

11. Y. Aharonov and D. Bohm, Phys. Rev. 115, 485 (1959).

12. I.A. Luk'yanchuk and Y. Kopelevich, Phys. Rev. Lett. 93, 166402 (2004).

13. H. Bateman and A. Erdelyi, Higher Transcendental Functions, Mc Graw-Hill Book Company, Inc, New-York, Toronto, London (1953), Vol. 1, Sec. 1.10.

14. R.G. Goodrich, D.L. Maslov, A.F. Hebard, J.L. Sarrao, D. Hall, and Z. Fisk, Phys. Rev. Lett. 89, 026401 (2002).

15. G.P. Mikitik and Yu.V. Sharlai, Phys. Rev. Lett. 93, 106403 (2004).

16. J.C. Slonzewski and P.R. Weiss, Phys. Rev. 109, 272 (1958).

17. J.W. McClure, Phys. Rev. 108, 612 (1957).

18. N.B. Brandt, S.M. Chudinov, and Ya.G. Ponomarev, Semimetals I. Graphite and its Compouds, Elsevier, Amsterdam (1988).

19. D.E. Soule, J.W. McClure, and L.B. Smith, Phys. Rev. 134, A453 (1964).

20. S.J. Williamson, S. Foner, and M.S. Dresselhaus, Phys. Rev. 140, A1429 (1965).
21. G.P. Mikitik and Yu.V. Sharlai, Phys. Rev. B73, 235112 (2006).

22. G.P. Mikitik and Yu.V. Sharlai, Zh. Eksp. Teor. Fiz. 114, 1375 (1998) [Sov. Phys. JETP 87, 747 (1998)].

23. A.J. Bennett and L.M. Falicov, Phys. Rev. 136, A998 (1964).

24. J.P. Van Dyke, J.W. McClure, and J.F. Doar, Phys. Rev. B1, 2511 (1970).

25. W.J. O'Sullivan and J.E. Schirber, Phys. Rev. 151, 484 (1966); ibid. 162, 519 (1967).

26. A.S. Joseph and W.L. Gordon, Phys. Rev. 126, 489 (1962).

27. E.S. Itskevich, A.N. Vonsovskii, and V.A. Sukhoparov, Zh. Eksp. i Teor. Fiz., Pis'ma v Redaktsiju 2, 67 (1965) [Sov. Phys.-JETP Lett. 2, 42 (1965)].

28. G.P. Mikitik and Yu.V. Sharlai, Phys. Rev. B65, 184426 (2002).

29. Note that the first harmonic of the magnetic susceptibility is proportional to $\cos (2 \pi \delta)$ [1], and hence the quantity $\delta$ reduced to the interval $0-0.5$ can be found in experiments. 